\title{
К ТЕОРИИ ИЗМЕРЕНИЯ ЗАВИСЯЩИХ ОТ ВРЕМЕНИ СПЕКТРОВ
}

Данная работа посвящена исследованию роли измерения в теории зависящих от времени (переходных) спектров резонансного вторичного свечения (РВC). Основная цель - выяснить, что изменяется, если временной затвор поместить перед спектральным прибором, а не за ним, как в рассматривавшейся до сих пор схеме $\left[{ }^{1-5}\right]$. Полезным методическим приемом, позволяющим удобно использовать полученные ранее формулы, является рассмотрение схемы с двумя временными затворами, т. е. ситуацию, когда ранее рассматривавшаяся схема измерения спектров $\left[{ }^{1-5}\right]$ дополнена временным затвором перед спектральным прибором. Это позволило рассмотреть также некоторые общие вопросы регистрации переходных спектров.

Прежняя схема измерения переходного спектра РВС содержала диспергирующую систему (спектральный прибор) и расположенный за ней точечный детектор фотонов, снабженный временным затвором $\left[^{1-5}\right]$ (эту схему в дальнейшем будем называть первой). В таком случае переходный спектр $I_{1}(\Omega, \eta, t)$, определяемый как скорость счета фотонов в момент времени $t+\tau_{0}\left(\tau_{0}-\right.$ время пролета фотонов от излучателя до счетчика) при настройке диспергирующей системы на частоту $\Omega$ со спектральным разрешением $\eta$, описывается следующей формулой $\left[{ }^{1}\right]$ :

$$
\begin{gathered}
I_{1}(\Omega, \eta, t)=\iint_{-\infty}^{t} d t_{1} d t^{\prime}{ }_{1} \exp \left[i \Omega\left(t^{\prime}{ }_{1}-t_{1}\right)\right] C\left(t-t_{1}, t-t^{\prime}{ }_{1}\right) \times \\
\times \int_{-\infty}^{t_{1}} d t_{2} \int_{-\infty}^{t_{1}} d t^{\prime}{ }_{2} a\left(t^{\prime}{ }_{1}-t_{1}, t^{\prime}{ }_{1}-t^{\prime}{ }_{2}, t_{1}-t_{2}\right) S\left(t_{2}, t^{\prime}{ }_{2}\right) .
\end{gathered}
$$

Здесь $a\left(t^{\prime}{ }_{1}-t_{1}, t^{\prime}{ }_{1}-t^{\prime}{ }_{2}, t_{1}-t_{2}\right)-$ трехвременная корреляционная функция вещества, $S\left(t_{2}, t_{2}^{\prime}\right)$ - корреляционная функция возбуждающего светового излучения, $C\left(t-t_{1}, t-t_{1}^{\prime}\right)-$ корреляционная функция регистрации. Функция $S\left(t_{2}, t_{2}^{\prime}\right)$ заметно отличается от нуля в области $\left|t_{2}\right| \sim\left|t^{\prime}{ }_{2}\right| \sim \Delta^{-1}$ ( $\Delta-$ спектральная ширина возбуждающего импульса), а функция $C\left(t-t_{1}, t-t_{1}^{\prime}\right)-$ в области $t-t_{1} \sim t-t_{1}{ }_{1} \sim \eta^{-1}$. Время $\Delta^{-1}$ в случае когерентного возбуждающего импульса определяет его длительность, а $\eta^{-1}$ есть характерное время когерентности волн, прошедших диспергирующую систему. Оно определяет интервал времени $\left(t-\eta^{-1}, t\right)$, в течение которого были излучены фотоны, зарегистрированные счетчиком в момент времени $t+\tau_{0}$. За начало отсчета времени принят момент прохождения центра максимумом возбуждающего светового импульса. 
Рассмотрим теперь схему измерения, содержащую два временных затвора: один расположен перед спектральным прибором (первый затвор) и другой - между спектральным прибором и счетчиком (второй затвор). Допуская произвольные законы временной зависимости пропускания света затворами, эту схему, очевидно, следует рассматривать как общую принципиальную схему измерения переходных спектров при использовании одиночного счетчика фотонов. Она содержит прежнюю схему с одним временным затвором, если принять длительность пропускания света первым затвором бесконечно большой. Другим предельным случаем является схема, содержащая временной затвор только перед спектральным прибором (см. ниже).

Для анализа ситуаций обратимся к формуле (1). Учтем, что переменные интегрирования $t_{1}$ и $t_{1}^{\prime}$ имеют смысл моментов переходов из промежуточного состояния в конечное в каждой из двух амплитуд соответственно. Временной затвор перед спектральным прибором выделяет определенную область из всей области возможных значений $t_{1}$ и $t_{1}^{\prime}$ между $-\infty$ и $t$, по которой проводится интегрирование. Поэтому его влияние можно описать функцкей временного пропускания $g\left(t_{1}, t_{h}^{\prime}\right)$, существенно отличной от нуля в области $\left|t_{1}-T\right| \sim\left|t^{\prime}{ }_{1}-T\right| \sim \Delta T$. Здесь $T+\tau_{0}^{\prime}$. среднее время пропускания света через первый затвор ( $\tau_{0}^{\prime}$ - время пролета фотона от излучателя до затвора), а $\Delta T-$ длительность пропускания. В результате формула для переходного спектра в рассматриваемом случае записывается в виде

$$
\begin{gathered}
I_{T, \Delta T}(\Omega, \eta, t)=\int_{-\infty}^{t} d t_{1} d t^{\prime}{ }_{1} \exp \left[\Omega\left(t_{1}^{\prime}-t_{1}\right)\right] g\left(t_{1}, t_{1}^{\prime}\right) C\left(t-t_{1}, t-t_{1}{ }_{1}\right) \times \\
\times \int_{-\infty}^{t_{1}} d t_{2} \int_{-\infty}^{t_{1}^{\prime}} d t^{\prime}{ }_{2} a\left(t^{\prime}{ }_{1}-t_{1}, t_{1}^{\prime}-t_{2}^{\prime}, t_{1}-t_{2}\right) S\left(t_{2}, t^{\prime}{ }_{2}\right) .
\end{gathered}
$$

Рассмотрим простой пример. Если временной затвор открыть в момент времени $T+\tau_{0}^{\prime}(T<t)$ и сразу же закрыть, то $g\left(t_{1}, t_{1}^{\prime}\right) \sim$ $\sim \delta\left(t_{1}-T\right) \delta\left(t_{1}^{\prime}-T\right)$ и

$$
I_{T, \Delta T}(\Omega, \eta, t)=C(t-T, t-T) \int_{-\infty}^{T} d t_{2} \int_{-\infty}^{T} d t^{\prime}{ }_{2} a\left(0, T-t_{2}^{\prime}, T-t_{2}\right) S\left(t_{2}, t_{2}^{\prime}\right),
$$

т. е. зависимость переходного спектра от частоты $\Omega$ пропадает. Этого и следовало ожидать, поскольку стоящий перед спектральным прибором $\delta$-образный временной затвор формирует белый спектр.

Определяемый формулой (2) спектр $I_{T, \Delta T}(\Omega, \eta, t)$ зависит от двух времен - $T$ и $t$. Интерес представляет также спектр, получаемый суммированием $I_{T, \Delta T}(\Omega, \eta, t)$ по всем моментам времени $t$ :

$$
I_{T, \Delta T}(\Omega, \eta) \equiv \int_{-\infty}^{\infty} d t I_{T, \Delta T}(\Omega, \eta, t)
$$

Этот спектр, как и спектр, измеренный по первой схеме, получается при использовании лишь одного временного затвора. Однако в данном случае затвор находится перед спектральным прибором. Такую экспериментальную схему в дальнейшем будем называть второй. Регистрируемый по этой схеме переходный спектр зависит от среднего времени $T$ и длительности пропускания света $\Delta T$ через затвор. Этот спектр, вообще говоря, зависит и от спектрального разрешения $\eta$. Учитывая, однако, что время регистрации и, тем самым, время прохождения спектрального прибора здесь не ограничено, можем всегда выбрать 
$\eta=0$ без ущерба временному разрешению. Тогда (см. Приложение)

$$
\begin{aligned}
& I_{T, \Delta T}(\Omega, 0) \equiv I_{2}(\Omega, \Delta T, T) \equiv \iint_{-\infty}^{\infty} d t_{1} d t^{\prime}{ }_{1} \exp \left[i \Omega\left(t^{\prime}{ }_{1}-t_{1}\right)\right] \times \\
& \times g\left(t_{1}, t^{\prime}{ }_{1}\right) \int_{-\infty}^{t_{1}} d t_{2} \int_{-\infty}^{t_{1}^{\prime}} d t^{\prime}{ }_{2} a\left(t^{\prime}{ }_{1}-t_{1}, t^{\prime}{ }_{1}-t^{\prime}{ }_{2}, t_{1}-t_{2}\right) S\left(t_{2}, t_{2}^{\prime}\right) .
\end{aligned}
$$

Этот переходный спектр, как и спектр $I_{1}(\Omega, \eta, t)$, зависит от трех переменных.

Рассмотрим, как влияет временной затвор перед спектральным прибором на измеряемый спектр на примере чисто электронной линии (ЧЭЛ) люминесценции прнмесного центра, возбуждаемой мгновенным световым импульсом и имеющей распадную ширину $\gamma$. В этом случае фигурирующие в (2) корреляционные функции имеют следующий вид:

$$
\begin{gathered}
S\left(t_{2}, t^{\prime}{ }_{2}\right) \sim \mid \delta\left(t_{2}\right) \delta\left(t^{\prime}{ }_{2}\right), \\
a\left(t^{\prime}{ }_{1}-t_{1}, t^{\prime}{ }_{1}, t_{1}\right) \sim \exp \left[i \Omega_{21}\left(t_{1}-t^{\prime}{ }_{1}\right)-\gamma / 2\left(t_{1}+t^{\prime}{ }_{1}\right)\right] .
\end{gathered}
$$

Корреляционные функции спектрального и временного пропускания возьмем для простоты в экспоненциальном виде

$$
\begin{aligned}
& C\left(t-t_{1}, t-t^{\prime}{ }_{1}\right) \sim \exp \left[-\eta\left(t-t_{1}\right)\right] \exp \left[-\eta\left(t-t^{\prime}{ }_{1}\right)\right], \\
& g\left(t_{1}, t_{1}{ }_{1}\right) \sim \exp \left[-\Delta T^{-1}\left|T-t_{1}\right|\right] \exp \left[-\Delta T^{-1}\left|T-t^{\prime}{ }_{1}\right|\right] .
\end{aligned}
$$

В этом случае в момент времени $T+\tau_{0}$ временной затвор пропускает максимально, причем с увеличением $\left|t_{1}-T\right|$ и $\left|t_{1}{ }_{1}-T\right|$ пропускание падает экспоненциально.

Подставим выражения (5)-(8) в (2). Получим следующую формулу для переходного спектра ЧЭЛ люминесценции:

$$
\begin{gathered}
I_{T, \Delta T}(\Omega, \eta, t) \sim \int_{0}^{t} \int d t_{1} d t^{\prime}{ }_{1} \exp \left[i\left(\Omega_{21}-\Omega\right)\left(t_{1}-t^{\prime}{ }_{1}\right)-\Delta T^{-1}\left|T-t_{1}\right|-\right. \\
\left.-\Delta T^{-1}\left|T-t^{\prime}{ }_{1}\right|-2 \eta t-(\gamma / 2-\eta)\left(t_{1}+t^{\prime}{ }_{1}\right)\right]
\end{gathered}
$$

или

$$
I_{T, \Delta T}(\Omega, \eta, t)=\left\{\begin{array}{l}
\exp (-2 \eta t-2 T / \Delta T)\left|\frac{1}{\alpha}[\exp (\alpha t)-1]\right|^{2}, \quad t<T, \\
\exp (-2 \eta t) \mid \frac{\exp (-T / \Delta T)}{\alpha}[\exp (\alpha T)-1]+ \\
+\left.\frac{\exp (T / \Delta T)}{\beta}[\exp (\beta t)-\exp (\beta T)]\right|^{2}, t>T,
\end{array}\right.
$$

где $\alpha=i\left(\Omega_{21}-\Omega\right)+\eta-\gamma / 2+\Delta T^{-1}, \quad \beta=i\left(\Omega_{21}-\Omega\right)+\eta-\gamma / 2-\Delta T^{-1}$.

Проанализируем подроб̆ее $I_{T, \Delta T}(\Omega, \eta)$ в случае высокого спектрального разрешения $(\eta=0)$ :

$$
\begin{gathered}
I_{2}(\Omega, \Delta T, T)=\left[\left(\Omega_{21}-\Omega\right)^{2}+\right. \\
\left.+\left(\gamma / 2-\Delta T^{-1}\right)^{2}\right]^{-1}\left|\left\{\exp (-T / \Delta T)+\frac{2 \Delta T^{-1} \exp \left[i\left(\Omega_{21}-\Omega\right) T-T \gamma / 2\right]}{i\left(\Omega_{21}-\Omega\right)-\left(\gamma / 2+\Delta T^{-1}\right)}\right\}\right|^{2} \cdot
\end{gathered}
$$

Сравним этот спектр с переходным спектром, рассчитанным в [ $\left.{ }^{1}\right]$ по формуле (1) с использованием корреляционных функций (5)-(7): 


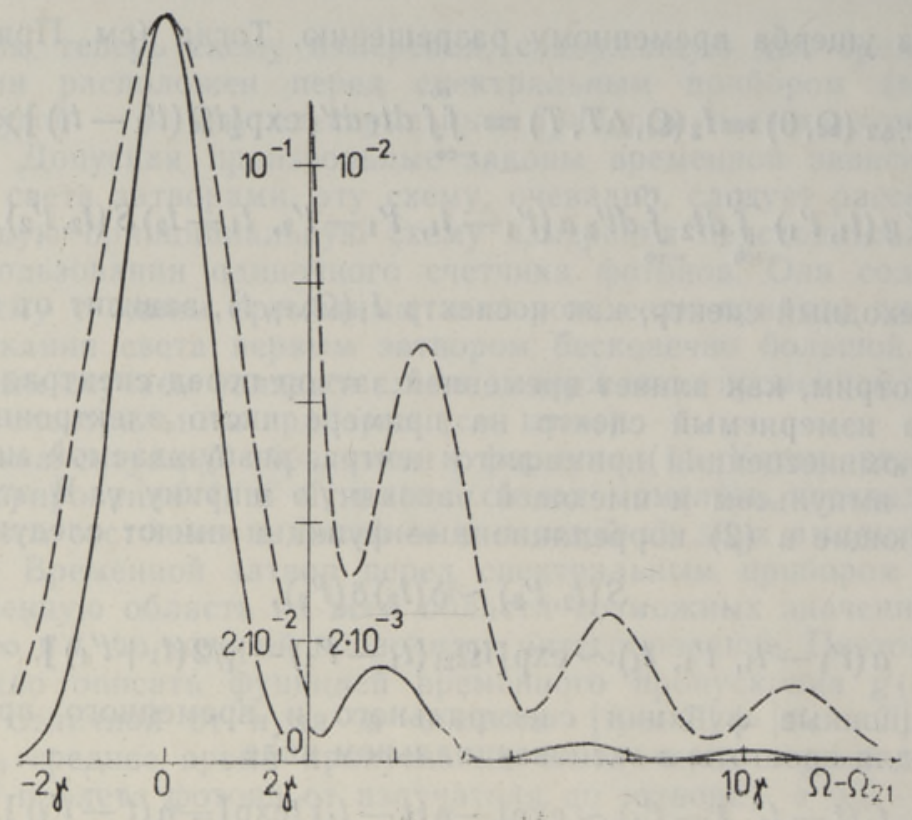

Рис. 1. Спектры $I_{2}(\Omega, \Delta T, T)$ (сп.лошная линия) и $I_{1}(\Omega, \eta, t)$ (прерывистая линия) при $T=t=(\gamma / 2)^{-1}$ и $\Delta T^{-1}=\eta=$ $=2(\gamma / 2)$.

$$
\begin{aligned}
I_{1}(\Omega, \eta, t)= & {\left[\left(\Omega_{21}-\Omega\right)^{2}+(\gamma / 2-\eta)^{2}\right]^{-1} \mid\{\exp (-\eta t)-} \\
& \left.-\exp \left[i\left(\Omega_{21}-\Omega\right) t-t \gamma / 2\right]\right\}\left.\right|^{2} .
\end{aligned}
$$

Спектр (12) (он был проанализирован в $\left.\left[{ }^{2,3}\right]\right)$ содержит осцилляции на крыльях контура. Зависимость ширины линии этого спектра от спектрального разрешения $\eta$ не монотонна. Кроме того, в этом спектре имеет место эффект компенсации распадной и регистрационной ширин, позволяющий при $\eta=\gamma / 2$ полностью исключить в переходном спектре распадную шкрину $\gamma$. Это дает возможность при достаточно больших $t$ получать линии с шириной, значительно меньшей как $\gamma$, так и $\eta$.

$\mathrm{Ha}$ рис. 1 в целях сравнения приведены спектры $I_{2}(\Omega, \Delta T, T)$ (см. (11)) и $I_{1}(\Omega, \eta, t)$ (см. (12)) в случае $\Delta T^{-1}=\eta$ и $T=t$. Видно, что спектр $I_{2}(\Omega, \Delta T, T)$ также имеет осцилляции на крыльях, хотя и более слабые.

$\mathrm{Ha}$ рис. 2 показаны результаты машинных расчетов зависимости полуширины (ширина линии на половине «высоты») $\sigma$ линий $I_{2}(\Omega, \Delta T, T)$ и $I_{11}(\Omega, \eta, t)$ соответственно от времен $T$ и $t(a)$ и параметров $\Delta T^{-1}$ и $\eta(\sigma)$. Видғо, что спектр $I_{2}(\Omega, \Delta T, T)$ монотонно сужается с ростом $T$ и может быть у́же $\gamma$ ( $\gamma$ на рис. 2 отмечена пунктиром). Существенно различаются обе схемы при $T=t=0$. В случае расположения временного затвора перед спектральным прибором, максимально пропускающим в начальный момент времени $T=0$ (вторая схема), к экспоненциально затухающей излучаемой линии $\exp \left(-\gamma / 2 \cdot t_{1}\right)$ добавляется экспоненциально падающее пропускание. В результате происходит замена $\gamma / 2$ на $(\gamma / 2)+\Delta T^{-1}$, т. е. ширина измеряемого спектра (11) при $T=0$ становится равной $\sigma=\gamma+2 \cdot \Delta T^{-1}$ (спектр измеряется во всем промежутке времени $t$ от 0 до $\infty)$. Для сравнения отметим, что в случае размещения временного затвора за спектральным прибо- 


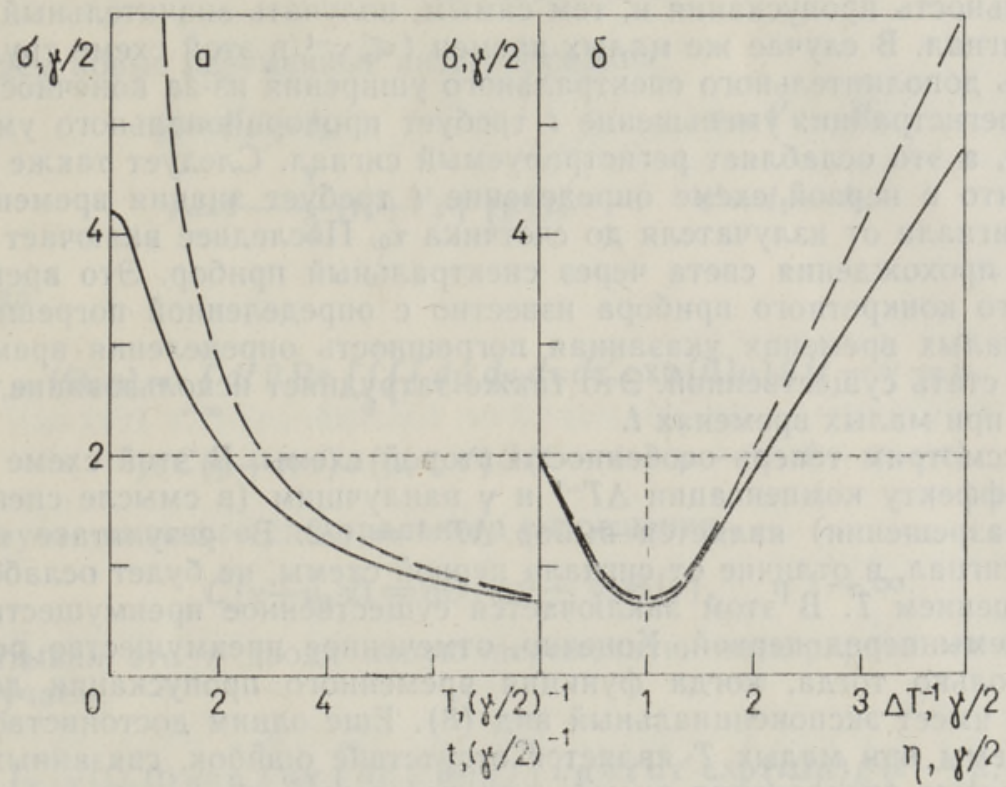

Рис. 2. Зависимость полуширины $\sigma$ спектров $I_{2}(\Omega, \Delta T, T$ ) (сплошная линия) и $I_{1}(\Omega, \eta, t)$ (прерывистая линия) от времен $T$ и $t$ при $\Delta T^{-1}=\eta=1,1(\gamma / 2)$ (a) и от $\Delta T^{-1}$ и $\eta$ при $T=t=8(\gamma / 2)^{-1}(б)$.

ром (первая схема) ширина спектра при $t=0$ бесконечна, поскольку как раз в момент $t=0$ происходит мгновенное возбуждение белым световым импульсом.

Зависимость ширины $I_{2}(\Omega, \Delta T, T)$ от параметра $\Delta T^{-1}$ не монотонна. При $\Delta T^{-1}=0$ (время пропускания бесконечно, т. е. временного затвора нет, — через спектральный прибор пропускается весь испущенный свет) полуширина линии $\sigma$ равна распадной ширине $\gamma$. При возрастании $\Delta T^{-1}$ (т. е. при уменьшении времени пропускания) ширина $\sigma$ вначале $\left(\Delta T^{-1}<\gamma / 2\right)$ уменьшается, а затем $\left(\Delta T^{-1}>\gamma / 2\right)$ растет. Мннимальное значение ширины линии (при $\Delta T^{-1}=\gamma / 2$ и при достаточно больших $T$ ) может быть существенно меныше $\gamma$ и $\Delta T^{-1}$. Действительно, при $\Delta T^{-1}=\gamma / 2$

$$
I_{2}(\Omega, \Delta T, T)=2 \mathrm{e}^{-\gamma T}\left[1-\cos \left(\left(\Omega_{21}-\Omega\right) T\right)\right] /\left(\Omega_{21}-\Omega\right)^{2},
$$

т. е. $\sigma \sim 1 / T \ll \gamma$ при $T \gg \gamma^{-1}$. Отметим также, что вклады $\Delta T^{-1}$ и $\eta$ в полуширину спектра $I_{T, \Delta T}(\Omega, \eta, t)$ не аддитивны.

Основываясь на полученных результатах, обсудим коротко особенности первой и второй схем регистрации.

Достоинством схемы с затвором после спектрального прибора является отсутствие дополнительного спектрального уширения при уменьшении длительности $\Delta t$ пропускания света через затвор. Это позволяет использовать достаточно малые $\Delta t$ и получать, в принципе, «мгновенные» спектры со сколь угодно узкими линиями. Следует, однако, учитывать, что вместе с уменьшением $\Delta t$ слабеет и регистрируемый сигнал. Поэтому целесообразно выбирать $\Delta t$ максимально большим, но не приводящим к заметному уширению спектра. При исследовании узких линий (с шириной $\sigma \sim t^{-1}$ ) для больших времен $t>\gamma^{-1}$ отмеченное уширение равно $\sim \Delta t / t^{2}$. Это позволяет использовать довольно большую 
длительность пропускания и, тем самым, получать значительный полезный сигнал. В случае же малых времен $t \leqslant \gamma^{-1}$ в этой схеме трудно избежать дополнительного спектрального уширения из-за конечности времени регистрации: уменьшение $t$ требует пропорционального уменьшения $\Delta t$, а это ослабляет регистрируемый сигнал. Следует также учитывать, что в первой схеме определение $t$ требует знания времени пролета сигнала от излучателя до счетчика $\tau_{0}$. Последнее включает в себя время прохождения света через спектральный прибор. Это время для каждого конкретного прибора известно с определенной погрешностью. При малых временах указанная погрешность определения времени $\tau_{0}$ может стать существенной. Это также затрудняет использование первой схемы при малых временах $t$.

Рассмотрим теперь особенности второй схемы. В этой схеме благодаря эффекту компенсации $\Delta T^{-1}$ и $\gamma$ наилучшим (в смысле спектрального разрешения) является выбор $\Delta T^{-1}=\gamma / 2$. В результате измеряемый сигнал, в отличне от сигнала первой схемы, не будет ослабевать с уменьшением $T$. В этом заключается существенное преимущество второй схемы перед первой. Конечно, отмеченное преимущество реализуется только тогда, когда функция временного пропускания действительно имеет экспоненциальный вид (8). Еще одним достоинством второй схемы при малых $T$ является отсутствне ошибок, связанных с погрешностью определения времени прохождения света через спектральный прибор. В области же больших времен вторая схема менее пригодна для получения узких линий, чем первая: длительность $\Delta T$ в этой схеме нельзя увеличивать пропорционально $T$, не теряя при этом эффекта компенсации (см. формулу (11)).

Таким образом, для регнстрации зависящих от времени спектров в области больших времен $t$ (в сравнении с характерными временами релаксации) целесообразно использовать рассмотренную ранее $\left[{ }^{1-3}\right]$ схему регистрации с временным затвором после спектрального прибора. В области же малых времен $t$ более подходящей оказывается рассмотренная в данной работе схема регистрации с временным затвором перед спектральным прибором.

В заключение отметим, что формулы (1) и (5), описывающие переходные спектры в первой и второй схемах измерения, переходят друг в друга, если принять

$$
C\left(t-t_{1}, t-t^{\prime}{ }_{1}\right)=\Theta\left(t-t_{1}\right) \Theta\left(t-t^{\prime}{ }_{1}\right) g\left(t_{1}, t_{1}^{\prime}\right) .
$$

В первой схеме измерения корреляционная функция спектрометра $C\left(t-t_{1}, t-t_{1}^{\prime}\right)$ задается лишь для положительных значений аргументов $t-t_{1}, t-t_{1}^{\prime}$. Во второй же схеме функция пропускания $g\left(t_{1}, t_{1}^{\prime}\right)$ задается на всей плоскости значений $t_{1}, t_{1}^{\prime}$. В этом смысле формула (5) является более общей, чем формула (1). С физической точки зрения это означает принципиальную возможность моделирования первой схемы второй.

Авторы признательны К. К. Ребане за предложение темы исследования и ценное обсуждение и А. Туулу за помощь в численных расчетах. 
ПРИЛОЖЕНИЕ

Введем новые переменные интегрирования:

$$
\begin{aligned}
& \mu=t_{1}^{\prime}-t_{1}, \\
& \tau=t_{1}^{\prime}-t_{2}^{\prime} \\
& v=t-\frac{1}{2}\left(t_{1}+t^{\prime}{ }_{1}+|\mu|\right), \quad \tau^{\prime}=t_{1}-t_{2} \text {. }
\end{aligned}
$$

Тогда

$$
\begin{gathered}
I_{T, \Delta T}(\Omega, \eta)=\int_{-\infty}^{\infty} d t 2 \operatorname{Re} \iiint_{0}^{\infty} \int d \mu d v d \tau d \tau^{\prime} \exp (i \Omega \mu) g(t-v-\mu, t-v) \times \\
\times C(\mu+v, v) a\left(\mu, \tau, \tau^{\prime}\right) S\left(t-v-\mu-\tau^{\prime}, t-v-\tau\right) .
\end{gathered}
$$

В случае высокого спектрального разрешения:

$$
C(v+\mu, v)=\eta \Theta\left(\eta^{-1}-v\right) \Theta(v), \quad \eta^{-1} \rightarrow \infty .
$$

Учитывая это и вводя новую переменную интегрирования $t^{\prime}=t-v$, получаем:

$$
\begin{gathered}
I_{T, \Delta T}(\Omega, 0)=\eta \int_{0}^{\eta^{-1}} d v \int_{-\infty}^{\infty} d t^{\prime} 2 \operatorname{Re} \iint_{0}^{\infty} \int d \mu d \tau d \tau^{\prime} \exp (i \Omega \mu) g\left(t^{\prime}-\mu, t^{\prime}\right) \times \\
\times a\left(\mu, \tau, \tau^{\prime}\right) S\left(t^{\prime}-\mu-\tau^{\prime}, t^{\prime}-\tau\right)
\end{gathered}
$$

или в переменных $t_{1}, t^{\prime}{ }_{1}, t_{2}, t_{2}^{\prime}$ :

$$
\begin{aligned}
I_{T, \Delta T}(\Omega, 0)= & \iint_{-\infty}^{\infty} d t_{1} d t^{\prime}{ }_{1} \int_{-\infty}^{t_{1}} d t_{2} \int_{-\infty}^{t_{1}^{\prime}} d t^{\prime}{ }_{2} \exp \left[i \Omega\left(t^{\prime}{ }_{1}-t_{1}\right)\right] g\left(t_{1}, t^{\prime}{ }_{1}\right) \times \\
& \times a\left(t^{\prime}{ }_{1}-t_{1}, t_{1}^{\prime}-t_{2}^{\prime}, t_{1}-t_{2}\right) S\left(t_{2}, t_{2}^{\prime}\right) .
\end{aligned}
$$

\section{Л И Т Е А Т У P A}

1. Хи жняков В. В.. Р ебане И. К., Ж. эксперим. и теор. физ., 74, вып. 3, 885896 (1978); Hizhnyakov, V., Technical Report of ISSR, Ser. A, № 860 (1977).

2. Ребане И. К., Ту ул А. Л., Х и жняков В. В., Ж. эксперим. и теор. физ., 77, вып. 4 (10), 1302-1312 (1979).

3. Реб ан И. К., Х и жняко в В. В., Изв. АН СССР, Сер. физ., 44, № 4, 854-858 (1980).

4. Eberly, J. H., Wodkiew icz, K., J. Opt. Soc. Amer., 67, № 9, 1252-1261 (1977).

5. S a a r i, P., ENSV TA Toimet. Füüs. Matem., 27, № 1, 109-111 (1978).
Институт физики
Академии наук Эстонской ССР
Поступила в редакцию 15/IX 1980

Inna REBANE, V. HIZNJAKOV

\section{AJAST SOLTUVATE SPEKTRITE MOOTTMISE TEOORIAST}

On leitud üldvalem ajast sõltuvate spektrite mõötmiseks skeemis, mis sisaldab kaks ajalist akent: üks enne ja teine pärast spektraalriista. Selline skeem sisaldab piirväärtustena kaht ühe ajalise aknaga skeemi: 1 , skeemis on ajaline aken pärast spektraalriista (varem 
vaadeldud juhtum), 2. skeemis enne spektraalriista, kusjuures viimase lahutusvöime on lōpmatult hea. Mõlemas skeemis on arvutatud puhtelektronjoone ajast sōltuv spekter. Mõlemas skeemis eksisteerib kompensatsiooniefekt: esimeses skeemis kompenseeruvad joone radiatsiooniline laius $\gamma$ ja spektraalriista lahutusvõime $\eta$, teises $\gamma$ ja akna lahtioleku aja pöördväärtus $\Delta T^{-1}$. Ajast sõltuvate spektrite registreerimiseks suurte aegade piirkonnas (võrreldes relaksatsiooniaegadega) on otstarbekam kasutada esimest skeemi, väikeste aegade piirkonnas teist skeemi.

Inna REBANE, V. HIZHNYAKOV

\section{ON THE THEORY OF MEASUREMENT OF TIME-DEPENDENT SPECTRA}

A scheme used for time-dependent (transient) spectra, which contains two time gates: one in front of and the other, behind the spectral device. Such scheme includes two cases of one-gate measurement: (i) time gate behind the spectral device (1st scheme, considered earlier), (ii) time gate in front of the spectral device.

The scheme considered earlier $\left[{ }^{1-5}\right]$ included a spectral device followed by pointlike photon detector 1 , which was supplied by a fast-operating time gate. The transient spectrum $I_{1}(\Omega, \eta, t)$ was determined as the photon counting rate at the moment $t$ with the spectral device tuned to the frequency $\Omega$ with the spectral resolution $\eta$ (1).

In the present scheme an additional time gate in front of the spectral device is used. This gate is described by two-time correlation function $g\left(t_{1}, t_{1}^{\prime}\right)$ characterized by the mean gating time $T$ and the non-zero duration of gating $\Delta T$. A general formula (2) is obtained describing a transient spectrum measured according to this scheme.

The following particular case is considered in detail: the recording scheme contains one time gate in front of the spectral device with high resolution $(\eta=0)$, the recordered spectrum is $I_{2}(\Omega, \Delta T, T)$ (the second scheme) (5). The spectrum $I_{2}(\Omega, \Delta T, T)$ of a purely electronic line of luminescence at an instantaneous excitation is compared with previously studied spectrum $I_{1}(\Omega, \eta, t)$ of the same line in the same model ((11), (12)).

It is shown that in both these spectra oscillation may occur on the wings of the contour (Fig. 1), although in the spectrum $I_{2}(\Omega, \Delta T, T)$ they are less manifest. Both spectra undergo narrowing with the growth of $t$ and $T$, respectively, and at a proper choice of parameters $\eta$ and $\Delta T^{-1}$ may become narrower than the decay width $\gamma$ (Fig. $2, a)$. In both schemes occurs the effect of the compensation of the decay width $\gamma$ and the apparatus "width": in the first scheme - a compensation of $\gamma$ and spectral resolution $\eta$, in the second one - that of $\gamma$ and reciprocal duration of gating $\Delta T^{-1}$ (Fig. 2, b).

In spite of the common features of $I_{1}(\Omega, \eta, t)$ and $I_{2}(\Omega, \Delta T, T)$, measured respectively by the first and second recording systems, these schemes are not of equal value. It is shown that for the recording of transient spectra in the region of long times (as compared to characteristic relaxation times) the first scheme is suitable, whereas the second one suits for short times. 